\title{
Mass spectrometry, Ribo-seq, and RNA-seq integration reveals hormone-dependent translation of short open reading frames in human long non-coding RNAs
}

\author{
Leonard Lipovich, Ph.D. \\ Professor, Cener for Molecular \\ Medicine and Genetic,s \\ Wayne State University \\ Detroit, Michigan, \\ United States of America \\ LLipovich@med.wayne.edu
}

\begin{abstract}
More than two-thirds of human genes do not encode proteins. Long noncoding RNA (IncRNA) genes are the most abundant class of non-protein-coding genes in humans, and their pivotal importance in both normal cellular functions and disease is becoming increasingly appreciated. These functions, rooted in RNA-driven biology independent of protein translation, have been amply documented. LncRNA genes are typically defined as those with all transcripts lacking any predicted Open Reading Frames (ORFs) over 100 amino acids in length and/or with homologies to known proteins. However, IncRNAs are often found in the cytoplasm, where, in principle, their short nonconserved ORFs - if any - may be translated by ribosomes, in a stochastic or perhaps a regulated fashion. Historically, two methods have been used to test whether IncRNAs are endogenously translated: direct discovery of IncRNA ORFs in protein mass spectrometry data (the original method; expensive, less common) and ribosome profiling (RiboSeq) (a newer approach, indirect, now commonly used). In the ENCODE (Encyclopedia of DNA Elements) Consortium, we were the first group to empirically test for IncRNA translation in human cells using mass spectrometry, finding that most IncRNAs are not translated. We have now deployed the two methods concurrently in a key nuclear hormone receptor pathway cancer model: human estrogen receptor positive MCF7
\end{abstract}

breast cancer cells before and after estrogen stimulation. Combining ribosome profiling with mass spectrometry, we identified four IncRNAs with short open reading frames that appear to be erroneously and persistently translated into unexpected peptides. One of these IncRNAs exhibits systematic in-frame mis-translation of multiple stop codons into amino acids, demonstrating an apparent gene-specific violation of the human genetic code. We also identified numerous estrogeninduced and estrogen-repressed IncRNAs jointly supported by Ribo-Seq and mass spectrometry, as well as estrogenresponsive differences in ribosome binding and translation that are distinct from estrogen-regulated transcriptional programs. Funding: National Institutes of Health - Director's New Innovator Award 1DP2-CA196375 (2014-2019) to L.L.

Keywords - long non-coding RNA, IncRNA, mass spectrometry, proteomics, cancer, human

Funding: National Institutes of Health - Director's New Innovator Award 1DP2-CA196375 (2014-2019) to L.L. 